\title{
On the Completeness of Three-Dimensional Electron Diffraction Data for Structural Analysis of Metal-Organic Frameworks
}

\author{
Meng Ge, ${ }^{\ddagger}$ Taimin Yang, ${ }^{\ddagger}$ Yanzhi Wang, ${ }^{\#}$ Francesco Carraro," Weibin Liang, ${ }^{\S}$ Christian Doonan, ${ }^{\S}$ \\ Paolo Falcaro," Haoquan Zheng, ${ }^{\#}$ Xiaodong Zou ${ }^{\ddagger}$ and Zhehao Huang ${ }^{\ddagger}, *$ \\ ${ }^{\ddagger}$ Department of Materials and Environmental Chemistry, Stockholm University, Stockholm SE-106 91, Sweden \\ "Key Laboratory of Applied Surface and Colloid Chemistry, Ministry of Education, School of Chemistry and \\ Chemical Engineering, Shaanxi Normal University, Xi’an 710119, China. \\ "Institute of Physical and Theoretical Chemistry, Graz University of Technology, Stremayrgasse 9, 8010 Graz, \\ Austria \\ ${ }^{\S}$ Department of Chemistry and the Centre for Advanced Nanomaterials, The University of Adelaide, Adelaide, 5005 \\ South Australia, Australia \\ E-mail: zhehao.huang@mmk.su.se.
}

\begin{abstract}
:
Three-dimensional electron diffraction (3DED) has been proven as an effective and accurate method for structure determination of nano-sized crystals. In the past decade, the crystal structures of various new complex metal-organic frameworks (MOFs) have been revealed by 3DED, which has been the key to understand their properties. However, due to the design of transmission electron microscopes (TEMs), one drawback of 3DED experiments is the limited tilt range of goniometer, which often leads to incomplete 3DED data, particularly when the crystal symmetry is low. This drawback can be overcome by high throughput data collection using continuous rotation electron diffraction (cRED), where data from a large number of crystals can be collected and merged. Here, we investigate the effects of improving completeness on structural analysis of MOFs. We use ZIF-EC1, a zeolitic imidazolate framework (ZIF), as an example. ZIF-EC1 crystallizes in a monoclinic system with a plate-like morphology. cRED data of ZIF-EC1 with different completeness and resolution were analyzed. The data completeness increased to $92.0 \%$ by merging ten datasets. Although the structures could be solved from individual datasets with a completeness as low as $44.5 \%$ and refined to a high precession (better than $0.04 \AA$ ), we demonstrate that a high data completeness could improve the structural model, especially on the electrostatic potential map. We further discuss the strategy adopted during data merging. We also show that ZIF-EC1 doped with cobalt can act as an efficient electrocatalyst for oxygen reduction reaction.
\end{abstract}




\section{Introduction}

During the past two decades, three-dimensional electron diffraction (3DED) has been steadily developed as a complementary technique to single-crystal X-ray diffraction (SCXRD) for structural elucidation of nanocrystals ${ }^{1-4}$. The early development of 3DED techniques such as $\mathrm{ADT} / \mathrm{PEDT}^{5,6}, \mathrm{RED}^{7,8}$, and $\mathrm{EDT}^{9}$ utilize stepwise rotation, where a crystal is tilted along an arbitrary axis in a transmission electron microscope (TEM), and electron diffraction (ED) patterns are collected at each angle. Recently, new protocols of 3DED, such as continuous rotation electron diffraction (cRED) $)^{10}$, fast $\mathrm{ADT}_{\mathrm{EDT}}{ }^{11,12}$, microED ${ }^{13}$, and rotation electron diffraction $^{14}$, have been developed. They are based on continuous data acquisition where the goniometer continuously rotates at a constant speed and the detector records a movie of ED patterns. The rapid development on 3DED has made large impacts in structural characterization of new materials, such as zeolites ${ }^{15-20}$, metal oxides ${ }^{21-24}$ and quasicrystal approximant ${ }^{25}$.

Metal-organic frameworks (MOFs) or porous coordination polymers (PCPs) are a class of hybrid materials by linking inorganic metal building units and organic ligands ${ }^{26,27}$. The almost unlimited combination of inorganic and organic components has led to an ever-expanding family of MOFs with versatile structures and properties ${ }^{28}$. However, due to the reversible coordination bonds, MOFs are sensitive to radiation damage by electron beam, which hampered their structural analysis using 3DED. This challenge has been tackled by the development of continuous rotation data collection, using which one 3DED dataset can be acquired in less than a few minutes, with a dose rate lower than 0.1 e s$^{-1} \AA^{-2}$. The fast data collection minimizes loss of crystallinity due to beam damage, and consequently the quality of 3DED data, such as resolution, has been improved significantly.

Benefited from continuous rotation electron diffraction, a growing number of MOF structures have been determined, with their unique properties revealed ${ }^{29-38}$. However, the geometric constraints in a TEM impose a physical limitation of the tilt range on the goniometer. Even using a specialized tomography sample holder, 3DED data can be acquired only from $-70^{\circ}$ to $+70^{\circ}$. The angular coverage of a maximum $140^{\circ}$ represents ca. $78 \%$ sampling volume of reciprocal space, where the remaining $22 \%$ of reciprocal space is not accessible. In practice, the tilt range is lower than the maximum value due to the movement of the target crystal, and also overlap of the target crystal with other crystals or TEM grid. The limited sampling therefore leads to an incomplete 3DED data, known as the missing cone problem. The incomplete data could hinder an accurate structure determination, which is more severe for MOF crystals with a low symmetry.

To solve this problem, Gruene et al. developed a specialized 3D TEM grid for 3DED data collection $^{39}$. As the data processing and structure determination using 3DED data are similar to those using SCXRD data, we present a strategy to improve data quality, particularly data completeness by merging data from different individual crystals. We use ZIF-EC1 as an example to studying the missing data problem. ZIF-EC1 was first discovered as a minor phase in a MOF mixture by continuous rotation electron diffraction (cRED). It crystallizes in a monoclinic space group $P 2_{1} / c$ with $a=13.462(2) \AA, b=14.659(3) \AA, c=14.449(2) \AA, \beta=118.12(1)^{\circ}$. The structure of ZIF-EC1 was first solved and refined using a single cRED data obtained from the phase mixture, 
and then using merged data from nine crystals of its pure phase ${ }^{40}$. Here we present a systematic study of the influence of data completeness on the structural analysis of ZIF-EC1. We show the data completeness can be improved after data merging. As each crystal has a different orientation and particle size, we further discuss the use of correlations of cRED intensity among different datasets for choosing the best combination to improve data quality. Moreover, merging of 3DED data is performed using different algorithms adapted from X-ray crystallography. We show the structure could be solved and refined to a high precision from individual datasets with a completeness as low as $44.5 \%$. With an increased data completeness, the structural model of ZIF-EC1 could be further improved, and peaks corresponding to atoms in the electrostatic potential map have well-defined isotropic shapes. By knowing the atomic structure and the underlying property, ZIF-EC1 was doped with cobalt and utilized as an electrocatalyst for oxygen reduction reaction (ORR).

\section{Experimental Section}

Synthesis. In a typical synthesis of ZIF-EC1, $0.125 \mathrm{~mL}$ of a $3.84 \mathrm{M}$ aqueous solution of 2methylimidazole (HmIM, TCI Chemicals) was mixed with $1.875 \mathrm{~mL}$ of deionized water. $1 \mathrm{~mL}$ of $0.24 \mathrm{M}$ aqueous solution of $\mathrm{Zn}(\mathrm{OAc}) 2 \cdot 2\left(\mathrm{H}_{2} \mathrm{O}\right)$ (EMSURE, Merck) was added into the above solution under vigorous stirring. The stirring condition was then maintained for at least $4 \mathrm{~h}$ at RT. The products were washed by deionized water for at least six times. After centrifugation, the products were re-suspended in deionized water. ZIF-EC1(Zn,Co) was synthesized in a modified condition. Instead of using $0.24 \mathrm{M}$ aqueous solution of $\mathrm{Zn}(\mathrm{OAc}) 2 \cdot 2\left(\mathrm{H}_{2} \mathrm{O}\right)$, a mixture of $0.16 \mathrm{M}$ $\mathrm{Zn}(\mathrm{OAc})_{2} \cdot 2\left(\mathrm{H}_{2} \mathrm{O}\right)$ and $0.08 \mathrm{M} \mathrm{Co}\left(\mathrm{NO}_{3}\right)_{2} \cdot 6\left(\mathrm{H}_{2} \mathrm{O}\right)$ was used, with a $\mathrm{Zn}$ :Co molar ratio of 2:1.

cRED data collection. The samples for cRED data collection were crushed in a mortar and dispersed in deionized water. A droplet was then taken from the suspension, put on a copper grid covered with lacey carbon, and dried in air at room temperature. cRED data were collected on a JEOL JEM2100 microscope operated at $200 \mathrm{kV}$ (Cs $1.0 \mathrm{~mm}$, point resolution $0.23 \mathrm{~nm}$ ). cRED data collection was controlled by using the data acquisition software Instamatic ${ }^{10,41}$, and the electron diffraction (ED) patterns were recorded by a Timepix hybrid detector QTPX-262k (512 $\times 512$ pixels, pixel size $55 \mu \mathrm{m}$, Amsterdam Sci. Ins.) A tomography holder was used for the data collection, which could tilt from $-70^{\circ}$ to $+70^{\circ}$ in the TEM. The area used for cRED data collection was about $1.0 \mu \mathrm{m}$ in diameter, as defined by a selected area aperture. To minimize electron beam damage of the crystal and maximize the data quality, data was collected at a low electron dose condition. Ten cRED datasets were collected on the ZIF-EC1 crystals, covering a tilt range from $42.34^{\circ}$ to $122.71^{\circ}$. Two different rotation speed were used for the datasets, and the data collection time ranges from 77.7 to 239.5 seconds. The detailed conditions for acquiring each dataset can be found in Table 1.

cRED data processing and structural analysis. The X-ray crystallography software package $X D S^{42}$ was used for data processing to determine the unit cell and space group, index the diffraction spots and estimate integrated diffraction intensities. XSCALE, which is part of the $X D S$ package, was then applied for data merging. The SHELX software package ${ }^{43}$ was used for structural analysis, where SHELXT was used for structure solution and SHELXL for structure refinement. Atomic scattering factors for electrons based on neutral atoms were used. All atoms 
were refined anisotropically. While the model obtained from each individual dataset could be refined, for easy comparison, the same starting SHELXL input file was used for all the structure refinements presented here. EADP were applied on two carbon atoms (C5 and C20). A planarity restraint (FLAT) was applied to the 2-methylimidazolate ( $\mathrm{mIm}^{-}$) groups to maintain a reasonable geometry. The similarity restraints as a combination of the free variables and the DFIX with DANG instruction were used to maintain reasonable C-C and C-N distances. At the end, EXTI was applied in the refinement. The crystallographic details can be found in Table 2.

Electrochemical application. All electrochemical tests are evaluated by a three-electrode system (The counter electrode and the reference electrode are graphite rods and saturated $\mathrm{Ag} / \mathrm{AgCl}$ electrodes, respectively) on the $\mathrm{CHI} 660 \mathrm{E}$ electrochemical workstation at $30{ }^{\circ} \mathrm{C}$. The working electrode is a rotating disk electrode (RDE) with a diameter of $5 \mathrm{~mm}\left(0.196 \mathrm{~cm}^{2}\right)$. CV test is carried out in $0.1 \mathrm{M} \mathrm{KOH}$ saturated with $\mathrm{Ar}$ or $\mathrm{O}_{2}$ (scan rate is $50 \mathrm{mV} \mathrm{s}^{-1}$ ). The linear sweep voltammetry. LSV test is carried out in $0.1 \mathrm{M} \mathrm{KOH}$ saturated with $\mathrm{O}_{2}$ (scan rate is $5 \mathrm{mV}$ $\mathrm{s}^{-1}$ ). The catalyst ink for ORR tests was prepared as following: $4 \mathrm{mg}$ ZIF-EC1(Zn, Co) and 1mg carbon nanotube was weighed and dispersed in mixed solution containing $450 \mu \mathrm{L}$ isopropanol, $40 \mu \mathrm{L}$ deionized water and $10 \mu \mathrm{L}$ Nafion D520 aqueous solution (5 wt\%). The mixed solution was treated by ultrasound for $1 \mathrm{~h}$ to form a homogeneous suspension. Then $15 \mu \mathrm{L}$ catalyst solution was taken from the pipette and uniformly dripped onto the rotating disc electrode (RDE) electrode. After drying, it was used for electrocatalytic ORR performance evaluation.

\section{Results and discussion}

ZIF-EC1 crystallizes in a monoclinic system with a plate-like morphology (Figure 1a). With a chemical formula of $\mathrm{Zn}_{3}(\mathrm{mIm})_{5}(\mathrm{OH})$, ZIF-EC1 is composed by $\mathrm{ZnN}_{4}$ mononuclear clusters and binuclear $\mathrm{Zn}_{2} \mathrm{~N}_{6}(\mathrm{OH})$ clusters, where $\mathrm{Zn}(\mathrm{II})$ cations link to $\mathrm{mIm}^{-}$ions (Figure 1b). Ten cRED datasets collected on the ZIF-EC1 crystals were chosen for this study (Table 1), among which eight (No. 1-8) are good datasets with high resolution (0.70-0.93 $\AA$ ) and another two (No. 9-10) with lower resolution (1.00-1.21 $\AA$ ). The 3D reciprocal lattices reconstructed from datasets 1-8 are presented in Figure 2, where the missing cones appear at different locations. As ZIF-EC1 is crystallized in a monoclinic system, the individual 3DED datasets have completeness ranging from $24.1-71.6 \%$ after the reflections were indexed by using X-ray crystallography software package $X D S^{42}$ (Table 1). The structure could be solved and refined from each of the datasets $\mathbf{1 -}$ 8.
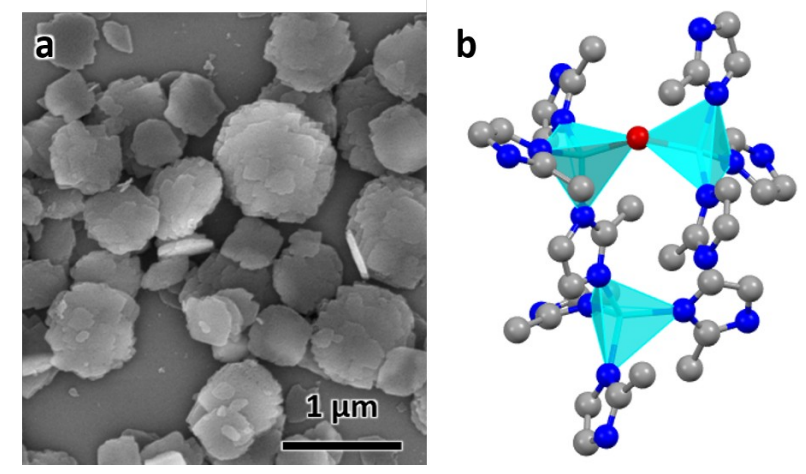
Figure 1. (a) SEM image of ZIF-EC1 crystals. (b) The structural model of ZIF-EC1. Cyan tetrahedra: Zn atoms; red spheres: $\mathrm{O}$ atoms; blue spheres: $\mathrm{N}$ atoms; grey spheres: $\mathrm{C}$ atoms.
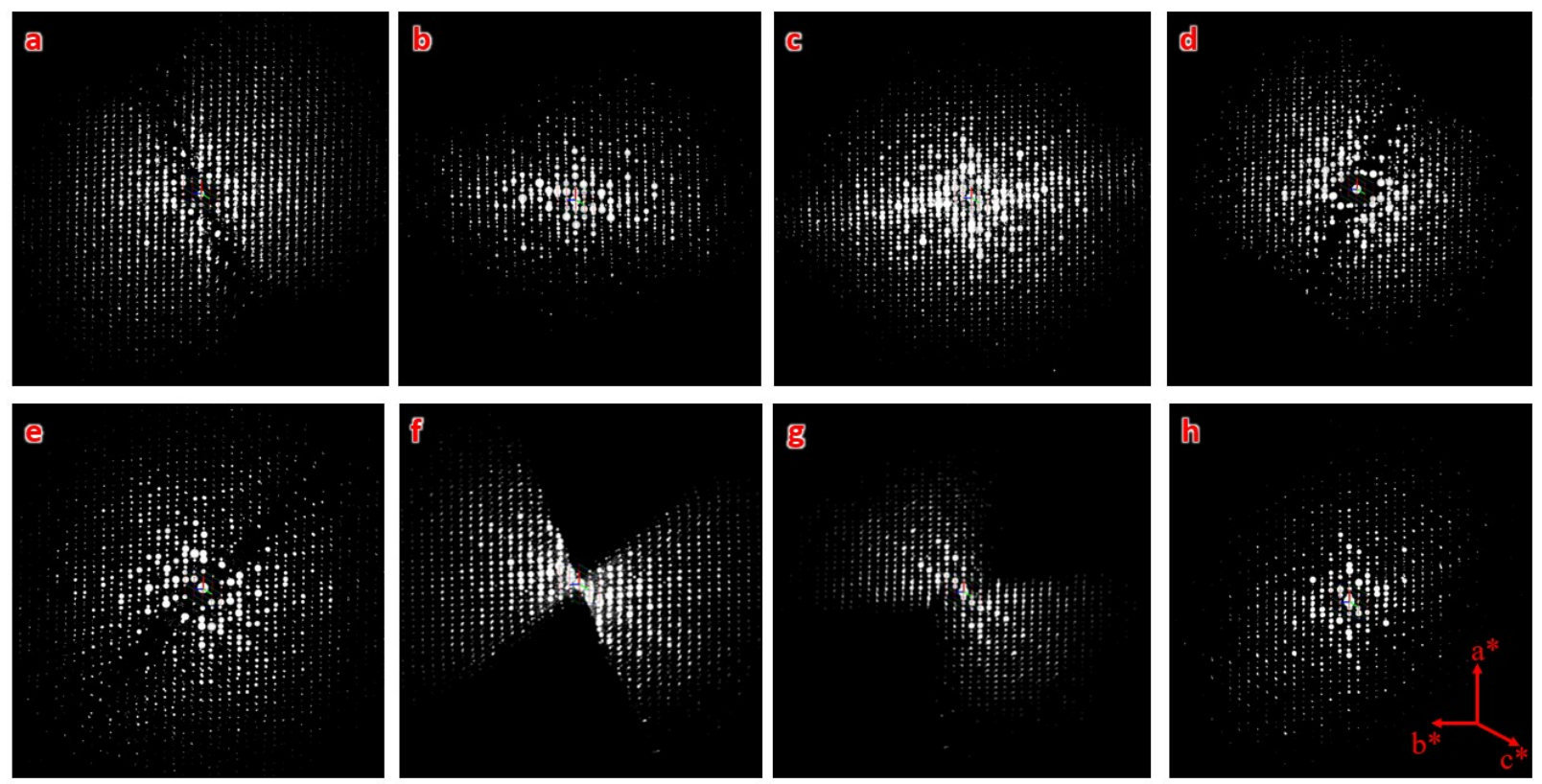

Figure 2. 3D reciprocal lattices of ZIF-EC1 reconstructed from the datasets (a-h) 1-8, respectively. They are viewed along the same direction, with data completeness of $44.5 \%, 47.1 \%, 55.0 \%, 58.3 \%, 52.9 \%$, $71.6 \%, 62.9 \%$, and $32.5 \%$, respectively. The different data cover different parts of the reciprocal space.

Table 1. Selected parameters and data information of ten cRED datasets collected on ZIF-EC1 crystals (200 kV, $\lambda=0.02508 \AA$ ).

\begin{tabular}{lcccccccccc}
\hline Dataset No. & $\mathbf{1}$ & $\mathbf{2}$ & $\mathbf{3}$ & $\mathbf{4}$ & $\mathbf{5}$ & $\mathbf{6}$ & $\mathbf{7}$ & $\mathbf{8}$ & $\mathbf{9}$ & $\mathbf{1 0}$ \\
Group & $\mathbf{A}$ & $\mathbf{A}$ & $\mathbf{A}$ & $\mathbf{A}$ & $\mathbf{B}$ & $\mathbf{B}$ & $\mathbf{C}$ & $\mathbf{C}$ & $\mathbf{D}$ & $\mathbf{D}$ \\
\hline Rotation Range $\left(^{\circ}\right)$ & 122.71 & 103.77 & 97.20 & 87.15 & 81.89 & 108.92 & 102.05 & 94.07 & 44.31 & 42.34 \\
Tilting Rate $\left({ }^{\circ} \mathrm{s}^{-1}\right)$ & 1.13 & 1.13 & 1.13 & 1.13 & 0.45 & 0.45 & 0.45 & 0.45 & 0.45 & 0.45 \\
Collection time (s) & 108.3 & 91.7 & 86.1 & 77.7 & 181.0 & 239.5 & 223.7 & 208.5 & 97.7 & 93.6 \\
Resolution $(\AA)$ & 0.70 & 0.78 & 0.75 & 0.74 & 0.76 & 0.78 & 0.93 & 0.92 & 1.21 & 1.00 \\
Completeness $(\%)$ & 44.5 & 47.1 & 55.0 & 58.3 & 52.9 & 71.6 & 62.9 & 32.5 & 40.8 & 24.1 \\
\hline
\end{tabular}

To improve the overall data quality, XSCALE was applied for data merging. We performed systematic studies by merging different number of datasets to study the influence of data completeness on the structural analysis of ZIF-EC1. We divided the ten datasets into four groups, collected under different tilting rates and/or with different resolutions. Group A includes four datasets 1-4 collected using a high tilting rate $\left(1.13^{\circ} \mathrm{s}^{-1}\right)$, all with high resolution $(0.70-$ $0.78 \AA$ ). The remaining datasets 5-10 were collected using a low tilting rate $\left(0.45^{\circ} \mathrm{s}^{-1}\right)$, groups according to the data resolution; datasets 5-6 with 0.76-0.78 $\AA$ resolution belong to Group $\mathbf{B}$, datasets 7-8 with 0.92-0.93 $\AA$ resolution to Group C, and datasets 9-10 with 1.00-1.21 $\AA$ resolution to Group D. We first merged datasets in Group A, which has completeness ranging from $44.5 \%$ to $58.3 \%$. The data completeness was improved steadily from $44.5 \%$ of the single 
dataset 1 to 71.5, 75.5, and 81.4\% for combining two (M_A1, Merged dataset A1), three (M_A2), and four datasets (M_A3), respectively (Table 2). To investigate the relationship between data completeness and the structural model, we refined the structural model of ZIF-EC1 against the data merged from different numbers of cRED datasets. While the number of unique reflections increased significantly, from 2189 for a single dataset $\mathbf{1}$ to 4154 by merging four datasets, the $R_{1}$ values are very similar, varying between 0.204 and 0.213 . This indicates that the refinement results keep improving. The improvement can be easily visualized in the corresponding electrostatic potential maps (Figures 3a-d and 3k-n). For those with high data completeness (75.5\% for M_A2 and 81.4\% for MA_3), the peaks appear more spherical with similar peak heights for the same atom types (Figures 3d and n). In contrast, data with low completeness (44.5\% for $\mathbf{1}$ and 62.6\% for M_A1) resulted in either missing peaks with large variations in peak heights (Figures 3a and k) or severe peak elongation (Figures 3a, b, k and l). Because the electrostatic potential map is the basis of a structural model, distortion of the map leads to reduced reliability and accuracy of the atom coordinates and atomic displacement parameters. Therefore, even though a structural model can be obtained from a single dataset, it is vital to improve data completeness.

Table 2. Refinement details of the merged datasets.

\begin{tabular}{|c|c|c|c|c|c|c|c|c|c|c|c|}
\hline Dataset no. & 1 & M_A1 & M_A2 & M_A3 & 5 & M_B & 7 & M_C & M_BC & M_ABC & $\begin{array}{c}\text { M_AB } \\
\text { CD }\end{array}$ \\
\hline $\begin{array}{l}\text { atasets used for } \\
\text { erging }\end{array}$ & N/A & $1 \& 2$ & $1-3$ & $1-4$ & N/A & $5 \& 6$ & N/A & $7 \& 8$ & $5-8$ & $1-8$ & $1-10$ \\
\hline Completeness (\%) & 44.5 & 71.5 & 75.5 & 81.4 & 53.5 & 85.0 & 62.9 & 63.3 & 87.0 & 89.1 & 92.0 \\
\hline & .78 & 78 & .78 & 0.78 & 0.78 & .78 & 0.93 & .93 & 0.78 & 0.78 & 0.78 \\
\hline feflections & 3935 & 9224 & 14352 & 19372 & 6502 & 16354 & 4958 & 7456 & 23817 & 43219 & 46079 \\
\hline $\begin{array}{l}\text { f reflections } \\
\text { ique) }\end{array}$ & 2189 & 3615 & 3848 & 4654 & 3132 & 4859 & 2088 & 2138 & 4976 & 5093 & 5262 \\
\hline $\begin{array}{l}\text { No. of reflections } \\
\text { I > }>2 \text { sigma(I)) }\end{array}$ & 1495 & 2420 & 2845 & 3306 & 1874 & 3263 & 1221 & 1409 & 3345 & 3933 & 3669 \\
\hline $\operatorname{sigma(I))}$ & 0.174 & 0.179 & 0.178 & 0.181 & 0.178 & 0.182 & 0.153 & 0.150 & 0.170 & 0.173 & 0.181 \\
\hline I reflections) & 0.204 & 0.213 & 0.204 & 0.211 & 0.219 & 0.211 & 0.193 & 0.181 & 0.201 & 0.193 & 0.250 \\
\hline Goof & 1.564 & 1.486 & 1.542 & & 1.870 & 1.442 & 1.321 & 1.409 & 1.450 & 1.676 & 1.530 \\
\hline $\operatorname{ADRA}(\AA) *$ & 0.035 & 0.027 & 0.018 & 0.015 & 0.038 & 0.015 & 0.036 & 0.034 & 0.010 & 0 & 0.012 \\
\hline
\end{tabular}

*The structural model refined against dataset M_ABC was used as the reference to calculate the ADRA values.

Furthermore, we applied data merging on datasets collected using a low tilting rate (Groups $\mathbf{B}$ and $\mathbf{C}$ ). The completeness increased significantly from $53.5 \%$ for single dataset $\mathbf{5}$ to 85.0\% with merging datasets 5 and $\mathbf{6}$ (M_B). However, there is little increase in data completeness for Group C, when datasets $\mathbf{7}$ and $\mathbf{8}$ are merged (from 62.9\% for $\mathbf{7}$ to 63.3\% for M_B). The electrostatic potential maps present a significant improvement from single dataset $\mathbf{5}$ to $\mathbf{M} \_$B as the completeness is increased from 53.5 to $85.0 \%$ (Figures 3f, g, p, and q). On the contrary, there is very limited improvement in the electrostatic potential maps from single dataset 7 to M_C, because the completeness was only increased by $0.4 \%$ (Figures $3 \mathrm{~h}$, i, r, and s). Notably, merging the four datasets $\mathbf{5 - 8}$ in Groups B and C (M_BC) led to improved 
completeness (87.0\%), and the electrostatic potential map (Figures $3 \mathrm{j}$ and t). Further merging all the aforementioned datasets 1-8 (M_ABC) resulted in the highest completeness (89.1\%), and thus the best electrostatic potential map with most spherical peaks (Figures 3e and o). The structural model refined against the dataset $\mathbf{M} \_\mathbf{A B C}$ is therefore used as a reference model, to which the atomic coordinates from the structural models obtained from datasets $\mathbf{M} \_\mathbf{A 3}, \mathbf{M} \_\mathbf{B}$, M_C and M_BC were compared. It shows that with a low completeness, e.g. $63.3 \%$ for M_C, the corresponding structural model resulted in higher deviations on atomic positions, compared to those from other datasets with high completeness (Table 3). We further calculated the average deviation from reference atoms (ADRA) ${ }^{44}$ values to compare the different models (Table 2). The ADRA values decreased successively from $0.035 \AA$ to $0.015 \AA$ by merging more and more datasets in Group A (M_A3). Improving completeness from 53.5 to $85.0 \%$ in Group B (M_B) also led to decreasing ADRA values from $0.038 \AA$ to $0.015 \AA$. One the other hand, the ADRA values showed little improvement by merging datasets $\mathbf{7}$ and $\mathbf{8}$ in Group C (M_C), varied from 0.036 to $0.034 \AA$, with a completeness of 62.9 and $63.3 \%$, respectively. Moreover, merging the four datasets 5-8 in Groups B and C (87.0\%, M_BC) led to a reduced ADRA value (0.010 $\AA$ ). Figure 4 shows a clear trend that the ADRA values are reduced with the increase of the data completeness as well as the increase of number of unique reflections.
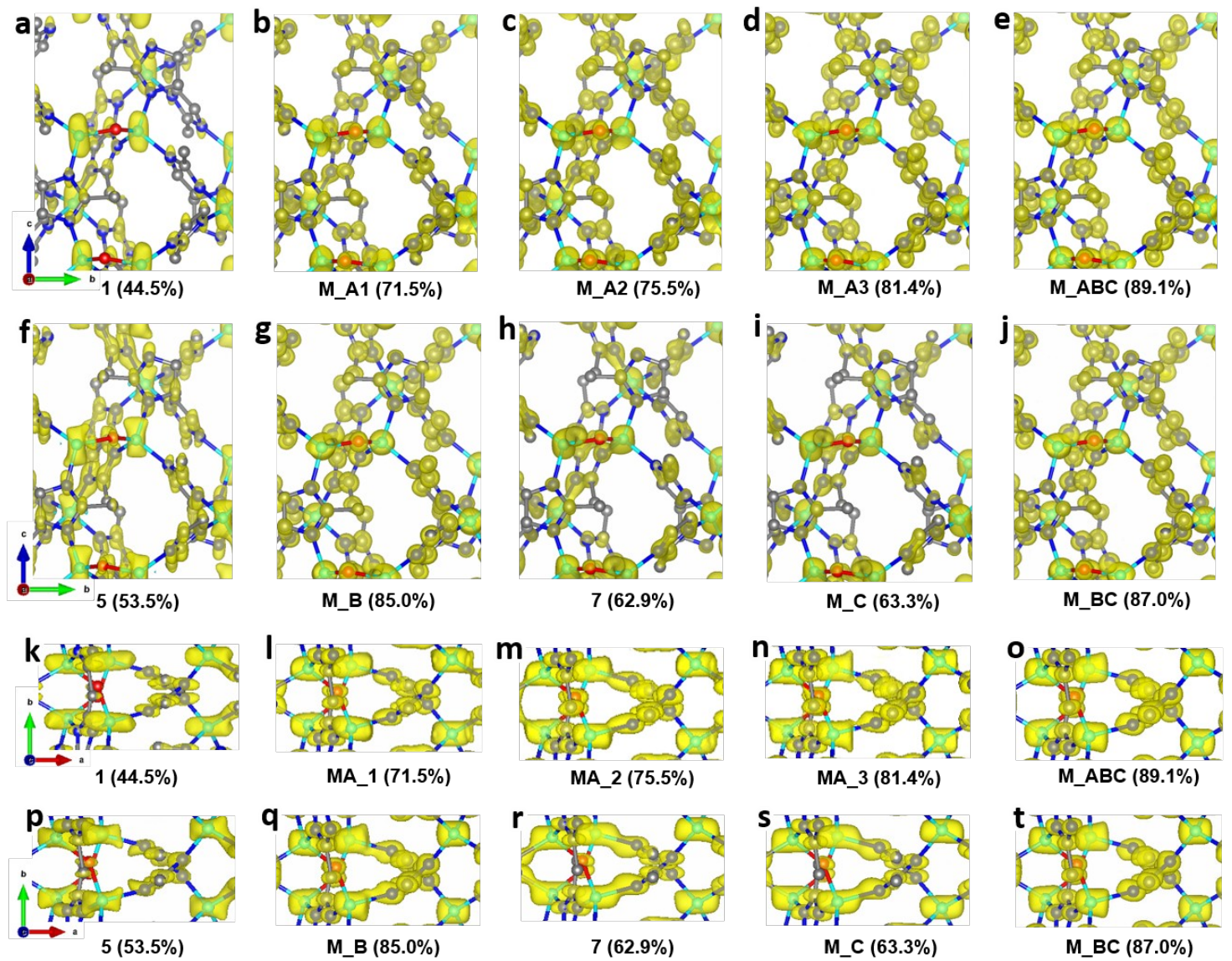

MA_3 (81.4\%)

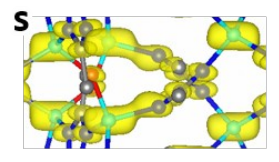

M_C (63.3\%)

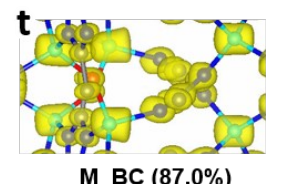

Figure 3. Electrostatic potential maps calculated based on datasets (a and k) 1, (b and l) MA_1, (c and m) MA_2, (d and n) MA_3, (e and o) M_ABC, (f and p) 5, (g and q) M_B, (h and r) 7, (i and s) M_C, and (j and t) M_BC, viewed along (a-j) the $a$-axis and $(\mathrm{k}-\mathrm{t})$ the $c$-axis. The refined structural model is 
superimposed in the map and used for calculating the structure factor phases. Missing peaks are observed on the maps constructed from data with low completeness. Peaks are more spherical with the increase of completeness. The electrostatic potential maps are drawn at the same contour level. Atom color codes: Grey: C; blue: N; red: O; cyan: Zn.

Table 3. Deviations of the atomic coordination. The structural model obtained from $\mathbf{M} \_\mathbf{A B C}$ is set as the reference.

\begin{tabular}{|c|c|c|c|c|}
\hline Atoms & M_A3 & M_B & M_C & M_BC \\
\hline Zn1 & $0.004(3)$ & $0.005(4)$ & $0.028(7)$ & $0.005(3)$ \\
\hline Zn2 & $0.002(4)$ & $0.005(5)$ & $0.031(8)$ & $0.003(4)$ \\
\hline Zn3 & $0.004(5)$ & $0.006(4)$ & $0.015(7)$ & $0.006(4)$ \\
\hline N1 & $0.008(15)$ & $0.014(12)$ & $0.030(21)$ & $0.014(10)$ \\
\hline N2 & $0.019(17)$ & $0.014(10)$ & $0.014(14)$ & $0.013(13)$ \\
\hline N3 & $0.019(17)$ & $0.027(11)$ & $0.014(17)$ & $0.021(12)$ \\
\hline N4 & $0.001(17)$ & $0.014(14)$ & $0.051(19)$ & $0.006(13)$ \\
\hline N5 & $0.007(12)$ & $0.013(14)$ & $0.045(20)$ & $0.003(11)$ \\
\hline N6 & $0.012(13)$ & $0.017(11)$ & $0.048(21)$ & $0.008(11)$ \\
\hline N7 & $0.012(10)$ & $0.010(14)$ & $0.069(17)$ & $0.015(12)$ \\
\hline N8 & $0.011(15)$ & $0.015(15)$ & $0.037(16)$ & $0.005(16)$ \\
\hline N9 & $0.008(13)$ & $0.008(15)$ & $0.040(18)$ & $0.005(11)$ \\
\hline N10 & $0.019(16)$ & $0.015(10)$ & $0.033(16)$ & $0.006(11)$ \\
\hline O1 & $0.025(13)$ & $0.015(17)$ & $0.048(22)$ & $0.010(18)$ \\
\hline C1 & $0.012(11)$ & $0.024(14)$ & $0.040(18)$ & $0.004(11)$ \\
\hline C2 & $0.013(17)$ & $0.006(12)$ & $0.024(17)$ & $0.004(12)$ \\
\hline C3 & $0.020(13)$ & $0.020(9)$ & $0.033(21)$ & $0.020(10)$ \\
\hline $\mathrm{C} 4$ & 0.007(13) & $0.003(14)$ & $0.037(23)$ & $0.010(15)$ \\
\hline C5 & $0.009(17)$ & $0.019(13)$ & $0.017(28)$ & $0.015(13)$ \\
\hline C6 & $0.030(19)$ & $0.033(17)$ & $0.041(20)$ & $0.018(16)$ \\
\hline C7 & $0.032(19)$ & $0.031(14)$ & $0.053(17)$ & $0.027(18)$ \\
\hline C8 & $0.034(15)$ & $0.004(14)$ & $0.073(29)$ & $0.019(13)$ \\
\hline C9 & $0.025(16)$ & $0.003(18)$ & $0.030(18)$ & $0.007(17)$ \\
\hline C10 & $0.015(15)$ & $0.015(14)$ & $0.041(21)$ & $0.010(12)$ \\
\hline C11 & $0.007(20)$ & $0.016(15)$ & $0.025(21)$ & $0.013(14)$ \\
\hline C12 & $0.010(16)$ & $0.017(18)$ & $0.022(19)$ & $0.008(16)$ \\
\hline C13 & $0.022(17)$ & $0.012(15)$ & $0.017(16)$ & $0.014(14)$ \\
\hline C14 & $0.029(16)$ & $0.009(14)$ & $0.034(26)$ & $0.008(14)$ \\
\hline C15 & $0.019(20)$ & $0.045(14)$ & $0.030(23)$ & $0.015(13)$ \\
\hline C16 & $0.007(14)$ & $0.006(18)$ & $0.016(28)$ & $0.005(19)$ \\
\hline C17 & $0.008(21)$ & $0.006(14)$ & $0.030(20)$ & $0.005(13)$ \\
\hline C18 & $0.019(17)$ & $0.027(21)$ & $0.028(7)$ & $0.008(20)$ \\
\hline C19 & $0.025(21)$ & $0.015(20)$ & $0.031(8)$ & $0.012(19)$ \\
\hline $\mathrm{C} 20$ & $0.022(16)$ & $0.027(16)$ & $0.015(7)$ & $0.006(14)$ \\
\hline
\end{tabular}

During data merging, correlation coefficients of the common reflection intensities $\left(\mathrm{CC}_{I}\right)$ between two datasets are calculated by XSCALE, which indicate the degree of intensity similarity between the datasets (Table 4). The correlation among the datasets 1-8 are very high, as indicated by the $\mathrm{CC}_{I}$ values ( $>0.95$ ). In contrast, datasets $\mathbf{9}$ and $\mathbf{1 0}$ are rather poorly correlated with the other datasets with the $\mathrm{CC}_{I}$ values are mostly below 0.80 . Combining the additional datasets 9 and $\mathbf{1 0}$ to the merged dataset $\mathbf{M}$ _ABC increased the data completeness from 89.1\% to $92.0 \%$. However, this led to a significant increase of the $R_{1}$ values for all reflections, from 0.193 
to 0.250 . This indicates that intensities of weak reflections are mostly affected. On the other hand, very little changes are observed in the electrostatic potential maps (Figure 5). Therefore, there would be little benefit to add more datasets with low intensity correlations, after a reasonable data completeness has already reached.
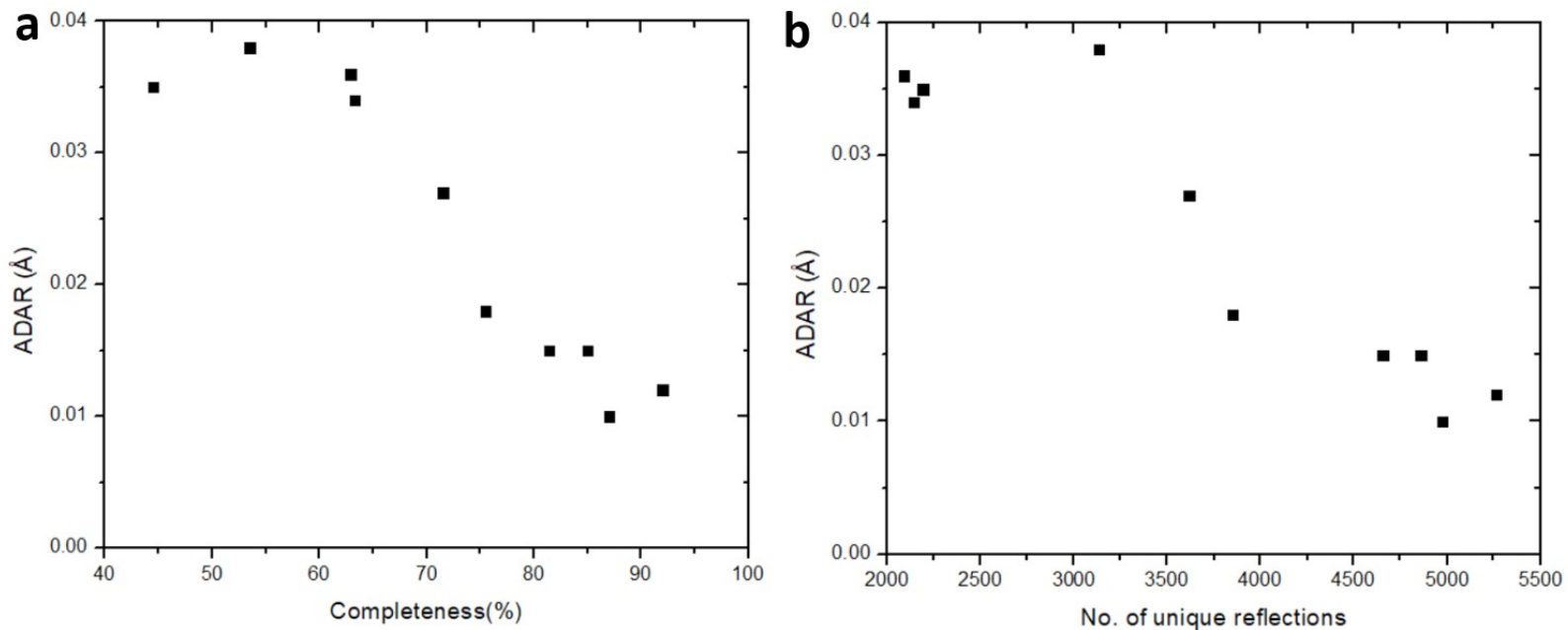

Figure 4. The effect of (a) data completeness and (b) number of total unique reflections on the ADRA values. The structural model refined against the merged data from the eight cRED datasets with the highest $\mathrm{CC}_{I}$ was used as the reference model. The ADRA values are reduced with the increase of the data completeness.

Table 4. The $\mathrm{CC}_{I}$ values between each two datasets.

\begin{tabular}{|c|c|c|c|c|c|c|c|}
\hline Datase & Dataset & No. of common & $\mathrm{CC}_{I}$ & Datase & Datas & No. of common & $\mathrm{CC}_{I}$ \\
\hline 1 & 2 & 2077 & 0973 & 3 & 8 & 1181 & 0979 \\
\hline 1 & 3 & 1572 & 0.967 & 4 & 8 & 944 & 0.983 \\
\hline 2 & 3 & 1323 & 0.964 & 5 & 8 & 870 & 0.985 \\
\hline 1 & 4 & 2904 & 0.986 & 6 & 8 & 1305 & 0.982 \\
\hline 2 & 4 & 1783 & 0.975 & 7 & 8 & 867 & 0.982 \\
\hline 3 & 4 & 1274 & 0.981 & 1 & 9 & 536 & 0.695 \\
\hline 1 & 5 & 2096 & 0.972 & 2 & 9 & 398 & 0.660 \\
\hline 2 & 5 & 1018 & 0.961 & 3 & 9 & 359 & 0.783 \\
\hline 3 & 5 & 1220 & 0.991 & 4 & 9 & 432 & 0.617 \\
\hline 4 & 5 & 1620 & 0.988 & 5 & 9 & 221 & 0.684 \\
\hline 1 & 6 & 2463 & 0.953 & 6 & 9 & 346 & 0.456 \\
\hline 2 & 6 & 1663 & 0.978 & 7 & 9 & 193 & 0.927 \\
\hline 3 & 6 & 1606 & 0.952 & 8 & 9 & 218 & 0.772 \\
\hline 4 & 6 & 1999 & 0.981 & 1 & 10 & 580 & 0.826 \\
\hline 5 & 6 & 1962 & 0.962 & 2 & 10 & 508 & 0.775 \\
\hline 1 & 7 & 684 & 0.961 & 3 & 10 & 592 & 0.856 \\
\hline 2 & 7 & 898 & 0.977 & 4 & 10 & 477 & 0.556 \\
\hline 3 & 7 & 934 & 0.995 & 5 & 10 & 410 & 0.722 \\
\hline 4 & 7 & 504 & 0.954 & 6 & 10 & 475 & 0.748 \\
\hline
\end{tabular}




\begin{tabular}{cccc|cccc}
5 & 7 & 484 & 0.990 & 7 & 10 & 312 & 0.369 \\
6 & 7 & 747 & 0.975 & 8 & 10 & 406 & 0.449 \\
1 & 8 & 1317 & 0.967 & 9 & 10 & 141 & 0.948 \\
2 & 8 & 1642 & 0.974 & & & & \\
\hline
\end{tabular}
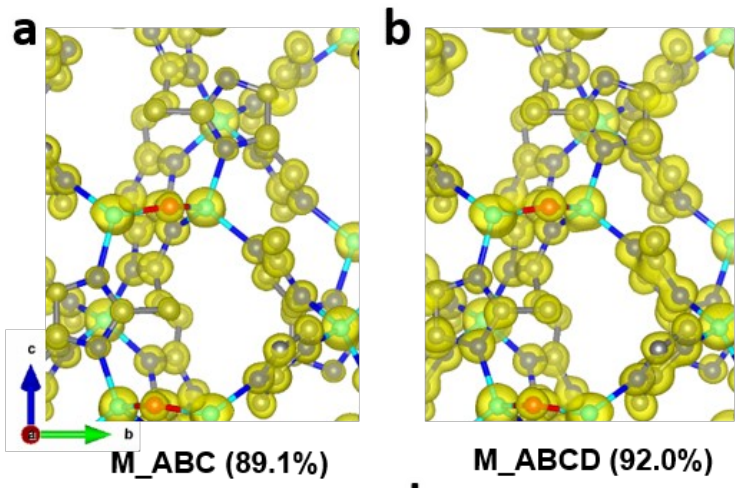

M_ABCD (92.0\%)
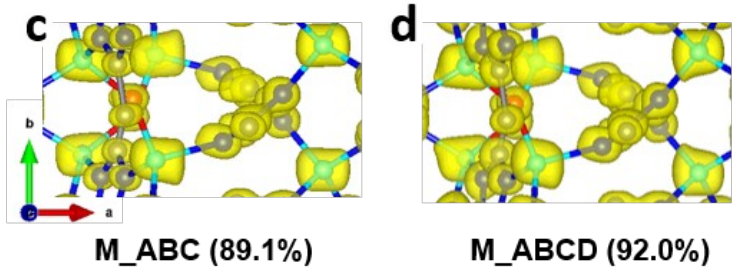

Figure 5. Electrostatic potential maps calculated based on datasets (a and c) M_ABC, and (b and d) M_ABCD viewed along (a and b) the $a$-axis and (c and d) the $c$-axis. The refined structural model is superimposed in the map and used for calculating the structure factor phases. The electrostatic potential maps are drawn at the same contour level. Atom color codes: Grey: C; blue: N; red: O; cyan: Zn.

Due to the development of continuous rotation setup for collecting cRED data, software for X-ray crystallography can be easily adapted to process 3DED data. Thus, we investigate different algorithms adapted from X-ray crystallography for data merging and evaluate the data quality. Besides XSCALE, XPREP ${ }^{45}$ and AIMLESS ${ }^{46}$ are among the other programs that are commonly applied for merging datasets. We chose datasets 1-8 for merging because they have a high correlation among each other. Using different algorithms for scaling and merging data, XSCALE, XPREP, and AIMLESS resulted in slightly different completeness of 89.1, 85.1, and $90.4 \%$, respectively (Table 5). The $R_{1}$ values are also similar (0.192-0.213), and the electrostatic potential maps exhibit well defined and spherical peaks (Figure 6). Although using kinematical assumption, this indicates that different merging algorithms in X-ray crystallography programs can be adapted to process cRED data for a high data completeness.

Table 5. Refinement details for merging datasets 1-8 using different methods.

\begin{tabular}{|c|c|c|c|}
\hline Software used for merging & $\begin{array}{l}\text { XSCAL } \\
E\end{array}$ & $\begin{array}{l}X P R E \\
P\end{array}$ & AIMLESS \\
\hline No. of reflections (all unique) & 5093 & 5046 & 5116 \\
\hline Completeness (\%) & 89.1 & 85.2 & 90.4 \\
\hline Resolution ( $\AA$ ) & 0.78 & 0.78 & 0.78 \\
\hline No. of reflections (I > 2 sigma(I)) & 3933 & 3964 & 3879 \\
\hline
\end{tabular}




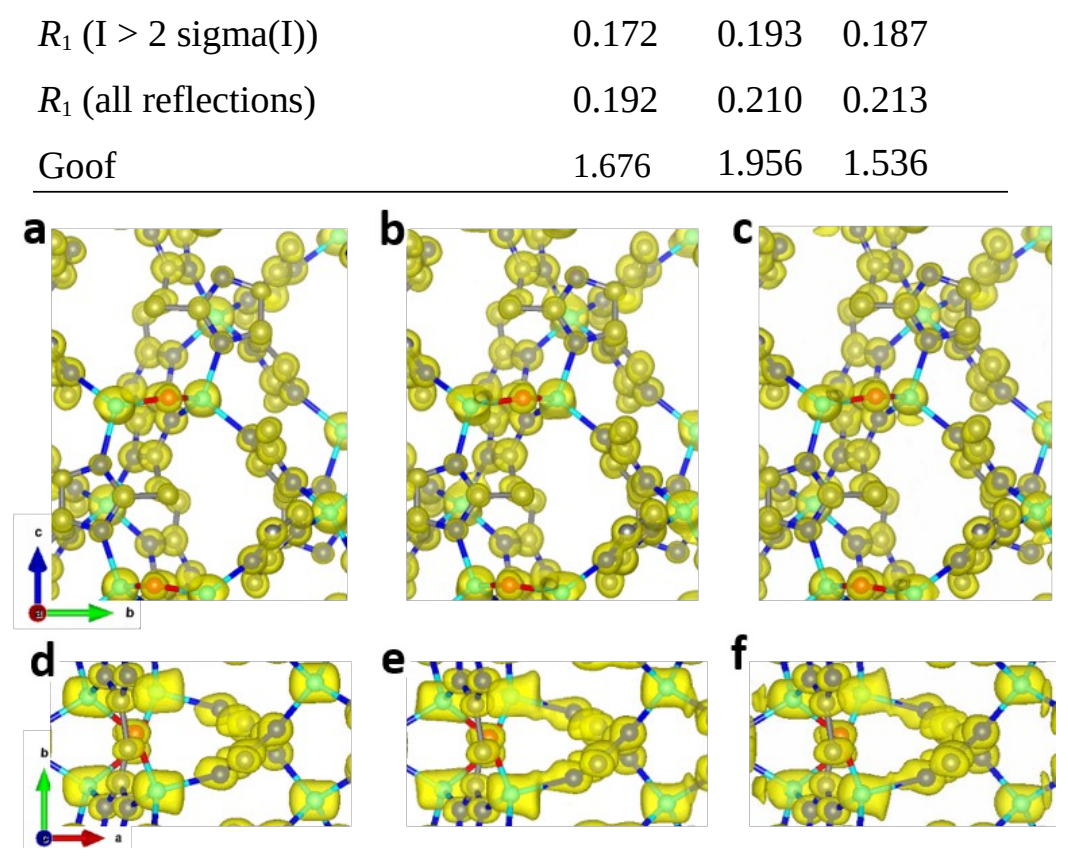

Figure 6. Electrostatic potential maps calculated based on datasets 1-8 merged by (a and c) XSCALE, (b and d) XPREP, and (c and e) AIMLESS. The refined structural model is superimposed in the map and used for calculating the structure factor phases. The electrostatic potential maps are drawn at the same contour level. Atom color codes: Grey: C; blue: N; red: O; cyan: Zn.

As 3DED revealed the accurate structure of ZIF-EC1, ZIF-EC1 has been utilized as precursor to generate carbon materials for catalyzing electrochemical reaction due to its high density of metal ions ${ }^{40}$. Here, we added Co(II) ions in the synthesis to further take advantage of the highly dense structure of ZIF-EC1, and use its pristine form for electrocatalytic oxygen reduction reaction (ORR). The electroactivity of ZIF-EC1(Zn,Co) was tested in $0.1 \mathrm{M} \mathrm{KOH}$ solution using rotating disk electrode (RDE). The cyclic voltammetry (CV) data of ZIFEC1(Zn,Co) showed that a strong electrocatalytic oxygen reduction peak near $0.74 \mathrm{~V}$ was observed in $\mathrm{O}_{2}$-saturated solution, while there was no reduction peak in Ar-saturated solution (Figure 7). Linear sweep voltammetry (LSV) further confirmed the good catalytic performance of ZIF-EC1, showing an onset potential ( $\left.E_{\text {onset }}\right)$ of $0.85 \mathrm{~V}$ and a half-wave potential $\left(\mathrm{E}_{1 / 2}\right)$ of 0.78 $\mathrm{V}$. In addition, the electrocatalytic ORR limiting current density can reach $4.7 \mathrm{~mA} \mathrm{~cm}^{-2}$. The efficiency of ZIF-EC1(Zn,Co) for ORR is among one of the best MOF electrocatalysts in comparison with the others ${ }^{47-50}$. 

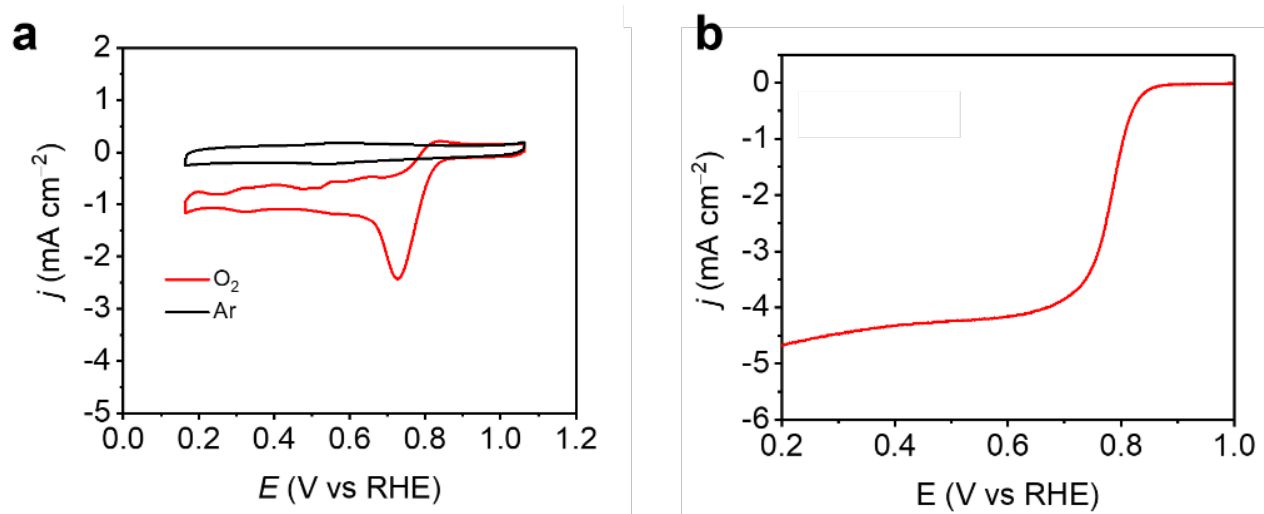

Figure 7. (a) CVs of ZIF-EC1(Zn,Co) catalyst in $0.1 \mathrm{M} \mathrm{KOH}$ by $\mathrm{Ar} / \mathrm{O}_{2}$-saturated. (b) LSV curves of ZIF$\mathrm{EC1}(\mathrm{Zn}, \mathrm{Co})$ catalyst in $0.1 \mathrm{M} \mathrm{KOH}$ by $\mathrm{O}_{2}$-saturated at $1600 \mathrm{rpm}$.

\section{Conclusions}

From the studies of the influence of data completeness on the structural analysis of ZIF-EC1, we show that the structure could be solved and refined to a high precision from cRED data with different completeness, even as low as $44.5 \%$ collected at various resolution (0.70-0.93 $\AA$ ). Importantly, we demonstrate that the completeness of 3DED data can be improved by merging data obtained from a series of individual crystals. The increased data completeness led to an improved structural model, which is indicated by the little changed $R_{1}$ values despite the increased number of unique reflections. In addition, the increased data completeness also resulted in decreasing of the ADRA values. More importantly, peaks corresponding to atom positions appear more spherical in the electrostatic potential maps, with similar peak heights for the same atom types. On the other hand, a low data completeness results in large uncertainty and variations in peak heights and elongation of the peaks. A completeness of $>75 \%$ already gave a low ADRA value $(<0.018 \AA$ ) and good electrostatic potential maps. The structural model may be further improved by merging more datasets, but only when the datasets with high intensity correlations (i.e. high $\mathrm{CC}_{I}$ values) are used. Data merging using different algorithms implemented in X-ray crystallographic software XSCALE, XPREP, and AIMLESS gave similar refinement results, showing the high quality of cRED data. Finally, we show that ZIF-EC1(Zn,Co) is an efficient electrocatalyst for ORR. With improved data quality, we anticipate that more structural details can be uncovered, which could promote discovery of novel properties of MOFs.

\section{Conflicts of interest}

There are no conflicts to declare.

\section{Supporting Information}

The crystallographic data for the datasets 1, M_A1, M_A2, M_A3, 5, M_B, 7, M_C, M_BC, M_ABC and M_ABCD have been deposited at the Cambridge Crystallographic Data Centre (CCDC, free for charge at https://www.ccdc.cam.ac.uk) under deposition number CCDC 2063943-2063953.

\section{Acknowledgements}


This work was supported by the Swedish Research Council (VR, 2016-04625, Z.H and 201704321, X.Z.), the Swedish Research Council Formas (2020-00831, Z.H.), and the National Natural Science Foundation of China (Grant No. 21975148, and 21601118).

\section{Notes and references}

1 M. Gemmi, E. Mugnaioli, T. E. Gorelik, U. Kolb, L. Palatinus, P. Boullay, S. Hovmöller and J. P. Abrahams, ACS Cent. Sci., 2019, 5, 1315-1329.

2 Z. Huang, E. S. Grape, J. Li, A. K. Inge and X. Zou, Coord. Chem. Rev., 2021, 427, 213583.

3 Z. Huang, T. Willhammar and X. Zou, Chem. Sci., 2021, 12, 1206-1219.

4 Z. Huang, M. Ge, F. Carraro, C. Doonan, P. Falcaro and X. Zou, Faraday Discuss., 2021, 225, $118-132$.

5 U. Kolb, T. Gorelik, C. Kübel, M. T. Otten and D. Hubert, Ultramicroscopy, 2007, 107, 507-513.

6 P. Boullay, L. Palatinus and N. Barrier, Inorg. Chem., 2013, 52, 6127-6135.

7 D. Zhang, P. Oleynikov, S. Hovmöller and X. Zou, Z Krist., 2010, 225, 94-102.

8 W. Wan, J. Sun, J. Su, S. Hovmöller and X. Zou, J. Appl. Crystallogr., 2013, 46, 1863-1873.

9 M. Gemmi and P. Oleynikov, Z. Für Krist. - Cryst. Mater., 2013, 228, 51-58.

10 M. O. Cichocka, J. Ångström, B. Wang, X. Zou and S. Smeets, J. Appl. Crystallogr., 2018, 51, $1652-1661$.

11 M. Gemmi, M. G. I. La Placa, A. S. Galanis, E. F. Rauch and S. Nicolopoulos, J. Appl. Crystallogr., 2015, 48, $718-727$.

12 S. Plana-Ruiz, Y. Krysiak, J. Portillo, E. Alig, S. Estradé, F. Peiró and U. Kolb, Ultramicroscopy, 2020, 211, 112951.

13 B. L. Nannenga, D. Shi, A. G. W. Leslie and T. Gonen, Nat. Methods, 2014, 11, 927-930.

14 I. Nederlof, E. van Genderen, Y.-W. Li and J. P. Abrahams, Acta Crystallogr. D Biol. Crystallogr., 2013, 69, 1223-1230.

15 J. Jiang, J. L. Jorda, J. Yu, L. A. Baumes, E. Mugnaioli, M. J. Diaz-Cabanas, U. Kolb and A. Corma, Science, 2011, 333, 1131-1134.

16 T. Willhammar, A. W. Burton, Y. Yun, J. Sun, M. Afeworki, K. G. Strohmaier, H. Vroman and X. Zou, J. Am. Chem. Soc., 2014, 136, 13570-13573.

17 J. Simancas, R. Simancas, P. J. Bereciartua, J. L. Jorda, F. Rey, A. Corma, S. Nicolopoulos, P. Pratim Das, M. Gemmi and E. Mugnaioli, J. Am. Chem. Soc., 2016, 138, 10116-10119.

18 P. Guo, J. Shin, A. G. Greenaway, J. G. Min, J. Su, H. J. Choi, L. Liu, P. A. Cox, S. B. Hong, P. A. Wright and X. Zou, Nature, 2015, 524, 74-78.

19 L. A. Villaescusa, J. Li, Z. Gao, J. Sun and M. A. Camblor, Angew. Chem., 2020, 132, 11379-11382.

20 Z. Huang, S. Seo, J. Shin, B. Wang, R. G. Bell, S. B. Hong and X. Zou, Nat. Commun., 2020, $11,3762$.

21 Y. Yun, W. Wan, F. Rabbani, J. Su, H. Xu, S. Hovmöller, M. Johnsson and X. Zou, J. Appl. Crystallogr., 2014, 47, 2048-2054.

22 E. Buixaderas, M. Kempa, V. Bovtun, C. Kadlec, M. Savinov, F. Borodavka, P. Vaněk, G. Steciuk, L. Palatinus and J. Dec, Phys. Rev. Mater., 2018, 2, 124402.

23 A. E. Lanza, M. Gemmi, L. Bindi, E. Mugnaioli and W. H. Paar, Acta Crystallogr. Sect. B Struct. Sci. Cryst. Eng. Mater., 2019, 75, 711-716.

24 J. Li, C. Lin, Y. Min, Y. Yuan, G. Li, S. Yang, P. Manuel, J. Lin and J. Sun, J. Am. Chem. Soc., 2019, 141, 4990-4996.

25 S. Samuha, E. Mugnaioli, B. Grushko, U. Kolb and L. Meshi, Acta Crystallogr. Sect. B Struct. Sci. Cryst. Eng. Mater., 2014, 70, 999-1005.

26 O. M. Yaghi, M. O’Keeffe, N. W. Ockwig, H. K. Chae, M. Eddaoudi and J. Kim, Nature, 2003, 423, 705-714.

27 S. Kitagawa, R. Kitaura and S. Noro, Angew. Chem. Int. Ed., 2004, 43, 2334-2375.

28 C. R. Groom, I. J. Bruno, M. P. Lightfoot and S. C. Ward, Acta Crystallogr. Sect. B Struct. Sci. Cryst. Eng. Mater., 2016, 72, 171-179.

29 M. Feyand, E. Mugnaioli, F. Vermoortele, B. Bueken, J. M. Dieterich, T. Reimer, U. Kolb, D. de Vos and N. Stock, Angew. Chem. Int. Ed., 2012, 51, 10373-10376.

30 N. Portolés-Gil, A. Lanza, N. Aliaga-Alcalde, J. A. Ayllón, M. Gemmi, E. Mugnaioli, A. M. López-Periago and C. Domingo, ACS Sustain. Chem. Eng., 2018, 6, 12309-12319.

31 S. Yuan, J.-S. Qin, H.-Q. Xu, J. Su, D. Rossi, Y. Chen, L. Zhang, C. Lollar, Q. Wang, H.-L. Jiang, D. H. Son, H. Xu, Z. Huang, X. Zou and H.-C. Zhou, ACS Cent. Sci., 2018, 4, 105-111. 
32 T. Rhauderwiek, H. Zhao, P. Hirschle, M. Döblinger, B. Bueken, H. Reinsch, D. D. Vos, S. Wuttke, U. Kolb and N. Stock, Chem. Sci., 2018, 9, 5467-5478.

33 B. Wang, T. Rhauderwiek, A. K. Inge, H. Xu, T. Yang, Z. Huang, N. Stock and X. Zou, Chem. - Eur. J., 2018, 24, 17429-17433.

34 E. S. Grape, J. G. Flores, T. Hidalgo, E. Martínez-Ahumada, A. Gutiérrez-Alejandre, A. Hautier, D. R. Williams, M. O’Keeffe, L. Öhrström, T. Willhammar, P. Horcajada, I. A. Ibarra and A. K. Inge, J. Am. Chem. Soc., 2020, 142, 16795-16804.

35 T. He, Z. Huang, S. Yuan, X.-L. Lv, X.-J. Kong, X. Zou, H.-C. Zhou and J.-R. Li, J. Am. Chem. Soc., 2020, 142, 13491-13499.

36 M. O. Cichocka, Z. Liang, D. Feng, S. Back, S. Siahrostami, X. Wang, L. Samperisi, Y. Sun, H. Xu, N. Hedin, H. Zheng, X. Zou, H.-C. Zhou and Z. Huang, J. Am. Chem. Soc., 2020, 142, 15386-15395.

37 F. Carraro, M. de J. Velásquez-Hernández, E. Astria, W. Liang, L. Twight, C. Parise, M. Ge, Z. Huang, R. Ricco, X. Zou, L. Villanova, C. O. Kappe, C. Doonan and P. Falcaro, Chem. Sci., 2020, 11, 3397-3404.

38 J.-H. Dou, M. Q. Arguilla, Y. Luo, J. Li, W. Zhang, L. Sun, J. L. Mancuso, L. Yang, T. Chen, L. R. Parent, G. Skorupskii, N. J. Libretto, C. Sun, M. C. Yang, P. V. Dip, E. J. Brignole, J. T. Miller, J. Kong, C. H. Hendon, J. Sun and M. Dincă, Nat. Mater., 2021, 20, 222-228.

39 J. T. C. Wennmacher, C. Zaubitzer, T. Li, Y. K. Bahk, J. Wang, J. A. van Bokhoven and T. Gruene, Nat. Commun., 2019, 10, 3316.

40 M. Ge, Y. Wang, F. Carraro, W. Liang, M. Roostaeinia, S. Siahrostami, D. M. Proserpio, C. Doonan, P. Falcaro, H. Zheng, X. Zou and Z. Huang, , DOI:10.26434/chemrxiv.13725817.

41 S. Smeets and W. Wan, J. Appl. Crystallogr., 2017, 50, 885-892.

42 W. Kabsch, Acta Crystallogr. D Biol. Crystallogr., 2010, 66, 125-132.

43 G. M. Sheldrick, Acta Crystallogr. A, 2008, 64, 112-122.

44 L. Palatinus, C. A. Corrêa, G. Steciuk, D. Jacob, P. Roussel, P. Boullay, M. Klementová, M. Gemmi, J. Kopeček, M. C. Domeneghetti, F. Cámara and V. Petříček, Acta Crystallogr. Sect. B Struct. Sci. Cryst. Eng. Mater., 2015, 71, 740-751.

45

46 P. R. Evans and G. N. Murshudov, Acta Crystallogr. D Biol. Crystallogr., 2013, 69, 1204-1214.

47 E. M. Miner, T. Fukushima, D. Sheberla, L. Sun, Y. Surendranath and M. Dincă, Nat. Commun., 2016, 7, 10942.

48 W. Cheng, X. Zhao, H. Su, F. Tang, W. Che, H. Zhang and Q. Liu, Nat. Energy, 2019, 4, 115-122.

49 E. M. Miner, S. Gul, N. D. Ricke, E. Pastor, J. Yano, V. K. Yachandra, T. Van Voorhis and M. Dincă, ACS Catal., 2017, 7, 7726-7731.

50 H. Zhong, K. H. Ly, M. Wang, Y. Krupskaya, X. Han, J. Zhang, J. Zhang, V. Kataev, B. Büchner, I. M. Weidinger, S. Kaskel, P. Liu, M. Chen, R. Dong and X. Feng, Angew. Chem. Int. Ed., 2019, 58, 10677-10682. 